In more advanced senility, characterized by retrograde changes in the venules and associated degenerations, and in which the arcus senilis is established, where the premonitory phenomena of apoplexy occur and are indicated by such marked symptoms as confusion of the intellect, incomplete aphasia with facial and other partial but transient palsies, the drug is, contrary to usual belief, indicated. This position is assumed after years of careful observation, with results that can not be disputed. The symptoms mentioned are due almost entirely to degenerative changes of the walls of the blood vessels. The vasomoter constrictors have failed in their function and that condition so essential to normality-circulatory equilibrium-is lost. The peculiarity of the venous system in having the capacity for containing the entire volume of blood in the body is here well shown, and the tension and pressure, always present in this condition, ruptures the altered radicals, as well as interferes with the phenomena of nutrition. The indications for treatment are plain, both from the mechanical and vital standpoint; restoration of the weakened heart, as far as possible, and especially of the vasomotor tone. This, this remedy accomplishes, and in so doing, the circulatory equilibrium is established and the distending forces upon the friable venules proportionately removed. Again, nutrition is rendered possible and life prolonged under the influences of the drug. Today there are living individuals who have enjoyed immunity for from one to three yeirs from symptoms which had long ago threatened destruction.

Another type of senility is constituted by albuminurics, who furnish examples of cyanotic kidney and who, with the usual concomitants of age, are additionally exhausted by the loss of albumin. This form of albuminuria greatly improves under the proper use of the remedy, and gain of flesh, strength and comfort is assured and pronounced. A typical instance is that of a gentleman, 67 years old, who, during the early summer of 1895 , suffered from an advanced state of this condition. The surcharged venous system was marked and the loss of albumin such as to engender great weakness. Disability from this latter was almost total and he was invalided. $\mathrm{He}$ has been, and is taking, thrice daily one-quarter grain doses, and notwithstanding the occurrence eleven months ago of sarcoma of the tonsil, which is treated by fragmental removal as occasion demands, he is so far bettered as to be able to comfortably engage in the lighter duties of every-day life. Fatty or primary degeneration of the cerebral cortex, if treated in the incipient stages, seems to be checked for considerable lengths of time.

The muscular weaknesses and vasomotor phenomena of those who recover almost entirely from hemiplegia except in these particulars and whose cardiac tone remains impaired, are much benefited.

Its use in overcoming the vasomotor element of shock and in proper cases of disease requiring capital surgical procedures, in which by reason of a poor circulatory system an anesthetic is dangerous, has been successfully tried in a limited number of cases, but sufficiently to prove its value. The circulatory crisis of acute disease, such for example as is encountered in acute lobar pneumonia, can be overcome, and the author has twice within the last few months so done by administering 2 grains of this alkaloid within two hours. Sufficient has been adduced to permit of the following conclusions:
1, Digitalin (German, pure, Merck) is a derivative of digitalis, not contaminated by other active principles; 2, it possesses a uniform and unvarying strength; 3 , it is relatively free from that property which produces gastric irritation; 4 , is a powerful stimulant to the whole cardiac apparatus; 5 , is a reliable and pronounced stimulant of the vasomotor system; 6, it does not develop cumulative action; 7, its adult dose ranges from the one-tenth grain as a minimum to one-half grain for a maximum.

\section{TRUE (IDIOPATHIC) ANGINA PECTORIS. ITS ETIOLOGY, PATHOLOGY AND} THERAPEUTICS.

Read by title in the Section on Practice of Medicine at the Forty eighth Annual Meeting of the American Medical Association, held at Philadelphia, Pa., June 1-4, 1897.

\section{BY THOS. L. MADDIN, A.M., M.D.}

PROFESSOR OF DISEASES OF THE NERVOUS SYSTEM AND GENERAL PATHOLOGY, MEDICAL DEPARTMENT, UNIVERSITY OF NASHYILLE, TENN.

Case 1.-Mr. E., a merchant from early manhood, habits excellent, possessed of a fine constitution, nover seriously sick, 60 years of age, consulted me in reference to a severe paroxysmal itching in his arms, vertigo, and torpid liver; these discomforts yielded to a simple line of treatment addressed to his liver, viz., frugality both in reference to the quantity and quality of his food, and much time in the open air. A few months afterward I was called to see him, in the early hours of the morning, on account of a severe and sudden paroxysm of bronchial and cardiac asthma. I found him almost cyanotic, with the most characteristic symptoms of asthma, and a scarcely perceptible and irregular pulse. The symptoms yielded readily after a hypodermic of morphia one-third gr. and atropia one hundred and twentieth gr. This was his first asthmatic parox. ysm and there was no history of it in his family ; he had only slight returns of it, but of ten from slight causes would have temporary palpitation of the heart, with profound disturbance of rhythm, but no evidence of organic disease. $\mathrm{He}$ also had great distress in the precordial region, agonizing pains over the left chest and down the arm. These paroxysms were traced to indigestion, worry, walking rapidly up hill, close confinement to office work, etc. I advised him to take a trip to California, be much in the open air, bo prudent and frugal in his food, and give special attention to his bowels and the state of his liver. I furnished him with a written description of his condition, my diagnosis of the pathology involved and therapeutic indica. tions for its treatment, so that in the event of having to call in medical aid, the physician could come to a quick knowledge of his needs. He first went to New York City and I advised him to call upon Dr. Austin Flint while there, show him my paper and request him to make a critical examination of his condition, especially of his heart, to see if there was any organic disease that I had failed to discover. A very thorough examination was afforded and Dr. Flint also had an opportunity to see my patient in one of his bad paroxysms; he endorsed the opinion expressed in my paper. Mr. E. was soon sufficiently relieved to start on his trip, was absent three months without any return of the paroxysm, and when he returned to Nashville had apparently recovered. He soon resumed his mercantile habits-close attention to the details of office business in an over-heated room, but little outdoor exercise, liberal indulgence in rich viands of the table, with recurrence of indigestion, paroxysms of palpitation and pectoral distress. I visited him one morning and found him cheerful and comfortable; he said that when seized with one of his spells the distress was so great he felt that one moment more would cause his death. This caused me such anxiety that $I$ made an unexpected visit at 9 P. M.; he answered the door in person, expressed surprise at seeing me and said he "had not felt so well for. several months." On my leaving he at once retired; his son occupied a bed in the same room and during the night was aroused by his father who remarked that he had gone into the library to see what time it was and found he had been sleeping two hours and was feeling so well he would not need any of the doctor's composing remedies. In about fifteen minutes his son thought he heard him move and raised up in bed to see, but as his father seemed to be asleep he gave himself no further concern. Early next morning the family servant came in, thought the old gentleman was very quiet, and found he was not only dead but rigor mortis was fully established. It was my opinion that 
he died when his son heard him move fifteen minutes after twelve o'clock, and that his death was instantaneous, for the covers of the bed were not disturbed.

Case 2.-.. Chancellor S., a man of fine constitution, but deli cate from studious habits, little exercise in the open air and torpid liver, was about 65 years of age; the pathology and symptoms of his sickness gave a clinical history parallel to that of Mr. E.; he died almost as suddenly. He had passed a quiet night, waked up at $4 \mathrm{~A}$. M., and was dead in less than fifteen minutes, of a paroxysm of angina pectoris. He was not the subject of any organic disease of the heart or blood vessels, but in my opinion was poisoned with the product of maldisintegration of the worn-out albumins of his own body -auto-intoxication.

Case 3.-Judge H., 55 years of age, had never been seriously sick, inherited a good constitution, was called upon to perform heavy and responsible duty under very discouraging circumstances: the needs in reference to his own health were neglected, his liver was torpid, bowels constipated, complexion sal low and mind depressed; after exposing himself one evening to inclement weather he was seized with a severe paroxysm of asthma-of bronchial tubes, diaphragm and heart-with in tense pain in the left chest, shoulder and arm, His condition was so critical that I administered, hypodermically, morphia one-third gr, and atropia one one-hundred and twentieth gr. this gave quick relief and he was soon asleep. There was no asthma afterward, but after three weeks he hac seizures of the heart, with all the symptoms of angina pectoris, and died very suddenly in one of them. There was no organic disease of the heart or arteries.

Case 4.-Mr. F., 50 years of age, is the subject of many par oxysms of angina pectoris; he has a delicate constitution, a sensitive nervous temperament, is intense in application to business and sleeps badly. His seizures come suddenly with intense precordial and substernal pains reaching to the left shoulder and to the elbow, and with a cord-like binding sensation around the chest, labored breathing, dry tongue and fauces, cold extremities, pale face, distressed expression, pulse scarcely to be detected, a sense of impending death, totally helpless, accumulation of gas in the stomach and intestines. He can not endure to be moved, and any imprudence in food emotional worry, or other strain upon the nervous system wil provoke a paroxysm. There is no organic disease in the heart or blood vessels.

Case 5.-Mr. G. has for many years been the subject of angina pectoris. The seizures are sudden and he falls whereever he may be ; can not be moved, is cold, pale, visage hag gard, pulse irregular, rapid and scarcely perceptible, great pre cordial distress extending to the left shoulder and elbow. The relief comes as suddenly as theseizure; sometimes the attack is short, at other times lasts three or four hours, or longer large quantities of gas in the stomach and intestines are always developed. $\mathrm{He}$ is torpid in his liver, while constipation and indigestion accompany a paroxysm. The exciting cause is imprudent indulgence in food.

The above cases furnish material for making the distinction between true and false angina pectoris; all systematic writers make this classification. Trousseau in his "Clinical Medicine," calls the true angina pectoris "idiopathic," the false "organic." We eliminate all organic disease of the heart and arteries from the discussion-fatty infiltration, fatty degeneration, stenosis of the gateways of the heart, valvular disease, hypertrophy and atrophy of the walls of the heart, dilatation or contraction of its cavities. atheromatous conditions of the heart or aorta, etc. These diseases may complicate true (idiopathic) angina pectoris and any further mention I make of them will be only as complications.

I claim that true (idiopathic) angina pectoris is a neurosis and may express itself in many forms-epilepsy of the heart, tetanus of the heart, asthma of the heart, chorea of the heart, syncope of the heart. The etiologic factor makes its impress upon the nerve centers that control the heart, arteries, capillaries and veins. The behavior of the muscles concerned in the circulation is determined by the exciting and immediate cause operating on the nerves and the nerve centers.
The etiology may be reflex from the alimentary canal, profound emotion either of pleasure or distress even from a dream; the most prolific cause is from auto-intoxication. We have in the manifold pathology included under the term uremia, a generic term given to the product of mal. disintegration of albuminoidsgout and rheumatism claim this form of paternity. When the kidney is afficted with Bright's disease, and fails to excrete or there is a failure of any other emunctory in its excretory duty, the blood is loaded with these impurities; they act as irritants to the nerve centers and develop many forms and degrees of ataxia-the coma vigil of fevers, subsultus tendinum and the different convulsions; many varieties of ataxia are expressed in the heart, arteries, capillaries and veins when the vaso-motor nervous system is irritated with these impurities. Ramborg says the abuse of tea, coffee, tobacco, etc., or reflex action from the stomach or intestine, uterine and ovarian disease, or great and continued strain of the mind or emotions, are at times the cause of angina pectoris. The great John Hunter was the subject and finally the victim of this disease. $\mathrm{He}$ was in the habit of saying that his life was suspended upon any emotional whim that might happen to him. Sedentary life in close confinement and overheated air, and too liberal indulgence in rich food are the thir rs that predispose to, and are often the exciting cause of angina pectoris; sometimes heredity is a factor.

We proceed now to analyze this neurotic disease: There are three essential factors pertaining to the nervous system concerned in the rhythmic muscular contraction of the heart-sympathetic, inhibitory and sensory (muscular-sensation); each of these is represented in maintaining physiologic equilibrium, not only of the heart but also of the arteries, capillaries and veins; it is through this nervous mechan. ism (nerve centers, nerves, ganglion and subsidiary nerve cells), that the katabolic force is dispensed and applied to the muscular tissue. According to the stimulus applied to the sensory factor, we will have a regular and normal rhythm, or some form of ataxia (a rapid or slow, a regular or irregular, high tension or low tension pulse), indeed all the modifications of the circulation are dependent upon and due to these nerve centers. In any case, when the sympatbetic nerve centers prevail and cause the death of the patient, the heart is contracted, is empty; its muscular tissue is in a state of tonic spasm (systole), the inhibitory centers are practically paralyzed (diastole), and from its similitude we may call it a cramp colic of the heart, the arteries or the capillaries, for it is irregular peristalsis and may lead to a tonic or clonic spasm and closure of their lumen; on the other hand, if the sympathetic nerve centers from whatever cruse are inadequate to balance the katabolism of the inhibitory nerve centers, the patient dies in diastole, with the heart cavities full of blood.

This want of physiologic harmony is often caused by auto-intoxication, a torpid liver and a failure of its disintegrating function in reducing the refuse albumin to urea; this establishes a diathetic condition which may show its presence under any trivial exciting cause, in a paroxysm of prurigo, asthma of the bronchial tubes, diaphragm, heart, ete.; all of these conditions are the offspring of the same parentage. When death is caused by angina pectoris the heurt may be in systole, diastole, or in statu quo, according to whether the sympathetic or inhibitory nerve cen- 
ters are affected, or both are alike impressed with the toxic cause, at the same time and to the same degree.

We give another path by which angina pectoris may be and often is caused; it is known that the heurt does not receive its nutrition directly from the blood passing through its cavities (auricles and ventricles), but that there is a special provision made for its nourishment- the coronary arteries, capillaries and veins; and the same system of centers, nerves and ganglia (sympathetic, inhibitory and sensory) determine their action. Any cause that broaks the harmony of these katabolic forces, will interfere with the blood supply for the nourishment of the heart tissue; every grade of ischemia or passive congestion may be present, nutrition be impoverished, potential or stored force fail and molecular and dynamic work be suspended; gradual fibroid stenosis of the walls of the coronary arteries (senility), regurgitation of the blood to the left ventricle (from valvular disease of the aortic opening), preventing the adequate supply of blood to the coronary arteries; spasm of the muscular walls of the coronary arteries may make a complete closure of their lumen, giving syncope of the heart. If the closure is only partial, nutrition fuils to the same degree, entailing a corresponding asthenia.

I wish here to demonstrate the nature of the homogenous substance of which the capillaries are made and claim that they have the function of contractility, which is a vital property, while elasticity is a mechanical and physical property. All muscular tissue is composed of sarcous cells and these are built of sarcous molecules, and each molecule is a muscle endowed with the property of contractility. Nutrition is the highest assimilating function and consists not only in storing matter, but also of force (potential). Euch molecule of a muscle is thus made vital with tonicity that is made manifest in contractility through metabolic disintegration. In order to realize this vital function it must be brought into relation with the nervous system, which is built up with nerve-cells made of nerve plasm, and this of molecules as its ultimate histologic analysis. We see and study muscular and nerve organs en musse, but when we analyze these two tissues in order to realize contractility, such as we have in the walls of the capillaries, the membrane of which it is made must consist of these two tissue molecules, viz., nerve and sarcous molecules. The nerve bioplasm, which forms a part of homogeneous membrane, is endowed with sensory, motor and inhibitory properties, precisely as we have claimed is due the nerve centers, nerves and ganglia which determine the rhythm of the heart and arteries.

If $I$ am correct in this claim, we may have a systemic capillary spasm closing their lumen, which may be partial or general, constituting a vis a fronte obstruction and damming the blood back into the arteries and heart. In this way we may have a peripheral or visceral condition that will realize one variety of angina pectoris. This peripheral high tension of arterioles and capillaries will bring the heart and large urteries to a standstill, overfilled with blood, in which case death may be immediate. It produces a pathology in the walls of the capillaries and arterioles that simulates an epileptic seizure of them, or the inhibitory nerve influence may prevail over the sympathetic and then the reverse condition will be present, or the sympathetic (systole) and inhibitory (diastole) may be impressed by the exciting cause at the same time and to the same degree; then we will have their walls in the condition of in statu quo-this simulates the cataleptic state.

What I claim is: That true (idiopathic) angina pectoris should be understood to be a generic term, covering many causes and many pathologies, but in every case it is a neurosis, in which the nervous system which determines and regulates harmony in the mechanism for the circulation of the blood, has been violated by some pathogenic cause. When we apply the above facts to the coronary arteries, arterioles and veins, we have another angina pectoris in which syncope is the pathologic factor. Syncope of an organ causes the suspension of the function of the organ; this is well illustrated in that form of aphasia (word blindness) in which the arteries, arterioles and capillaries supplied to the supra-orbital convolution of the brain are seized with a tonic spasm, and when it is relaxed and blood is again supplied to them, their syncope is relieved and their function is reëstablished. The same behavior of nerve forces (sympathetic, inhibitory and in statu quo) which I have mentioned in other forms of angina pectoris, pertain to the coronary arteries, capillaries and veins. When the spasm of the coronary blood vessels is relieved, by whatever cause, and the blood again flows through them, the heart resumes its function just as we have seen in paroxysmal aphasia. The fibroid change in the walls of the coronary blood vessels in the senile heart, which is one of the inheritances of age, leads to a progressive diminution of their lumen and to a gradual ischemia, that leads to a formidable complication of idiopathic angina pectoris, but this fibrosis of senility is not strictly an organic disease; as its genesis is physiologic, we will reckon it as making the heart more vulnerable to the causes of idiopathic angina pectoris. Porter has recorded experimental investigation in closure of coronary arteries of dogs. He found that complete closure was followed by arrest of the heart pulsations; but if one artery or a branch of it was closed, then there was irregular action or cessation of function, corresponding to the region of ischemiathe branches of the coronary arteries are physiologic, if no $t$ anatomic, terminal branches; on removing the obstruction the normal action of the heart was restored. Résumé.-Angina pectoris, false and true:

1. The false - all forms of organic diseases of heart, arteries, capillaries and veins.

2. The true-idiopathic (functional).

3. The blended type--when the organic is a complication of the idiopathic.

4. The true angina pectoris is a neurosis and may express itself in many forms.

5. The nervous mechanism involved contains three physiologic factors: $a$. Sympathetic centers and nerves - systolic (efferent). $b$ Inhibitory centers and nerves (sp. accessor)-diastolic (efferent). c. Sensory centers and nerves (vagus)-muscular-sensation (afferent). 6. Each of these physiologic factors is represented in the vaso-motor system of the centers, ganglia and nerves of the heart, arteries, capillaries and veins, and their physiologic equation determines the rhythmic harmony of the circulation in health.

7. From a chronic cause, a diathetic condition which establishes a vulnerability in the nervous system is produced-as in the mal-disintegration of nitrogen compounds and their failure of excretion, or some error in the product of the ductless glands, etc.-Which on the action of some exciting cause will determine an explosive paroxysm of systole, or of diastole, or of 
both at the same time, locking the sarcous substance in statu quo; this may be of the heart, arteries or capillaries, or of all at the same time; and in many cases the diaphragm and bronchial tubes are similarly impressed.

8. Symptoms of sensation: breast pang, dyspnea of the heart, sudden onset of precordial agony, pain extending from the heart to the left chest, shoulder and arm, sometimes to the right chest and shoulder; constriction of chest, an overpowering sense of impending death (all movements are dreaded lest they may precipitate that result), cold skin, deadly pallor, tension of arterial walls so that the pulse is arhythmic, diminished in strength and volume; the paroxysm often lasts a few seconds, minutes or sometimes is protracted; in many cases eructation of gas is present and often a quantity of pale urine is passed.

Treatment.-This is properly divided into three indications: 1. Curative-to treat the predisposing cause (the diathesis). 2. Preventive-to avoid all axciting causes. 3. Emergent-to relieve the immediate paroxysm.

1. Study the conditions that have produced the diathesis-heredity, bad habits and environments, but especially the quality and quantity of the food, and fortify the patient against them.

The most fertile cause of this diathesis is the quantity of fulminating food-that which is rich in nitrogen; therefore, it is best to refrain from the use of a meat diet; fruits are especially indicated, and much outdoor air, change of climate, pleasant companionship, etc.; in cases having any suspicion of a syphilitic -lement, bichlorid of mercury and iodid of potassium are indicated; these remedies, also Fowler's solution of arsenic are of curative value in many cases of autointoxication from errors of metabolism and the products of the ductless glands.

2. The second indication of treatment is to avoid the exciting cause of the paroxysm (indigestion, torpor of the liver, constipation, auto-infection from the intestines), and look especially after the disintegrating function of the liver, in order that its metabolism of nitrogeneous compounds may be reduced to ureathe physiologic diuretic; avoid physical strain, mental strain, emotional strain, and be discrete in personal habits and hygiene; be much in the open air, with invigorating environments and pleasant social contact; ropetition of physical, mental or emotional activity begets automatism; therefore, vigilance with reference to these physiologic habits should be practiced, before they have out grooves of activity into the nervous system.

3. The third indication of treatment is to relieve the patient from a paroxysm. The remedies do not aim to modify structure but to influence the function of the vaso-motor nervous mechanism. The following catalogue is a partial list of the medicines from which the physician selects: morphia, atropia, digitalis, strychnin, strophanthus, methylene-blue, veratrum viride, nitro-glycerin, nitrite of amyl, nitrite of sodium, hyoscin hydrobromate, etc., to be administered hypodermically when the emergency is great or by inhalation when practical.

We have in this list drugs that are prominent in their therapeutic virtues, from three points of viewsystolic, diastolic and analgesic. Digitalis is eminently fitted to improve tonicity, regulate the rhythm of the heart, arteries and capillaries; it acts alike on the norve mochanism, both of systole and diastole, it pro- tracts the time of rest in the events of a rhythm and thus promotes the nutrition of their walls. But it is not safe to give it until the tonic spasm is relaxed, whether it is in the heart, arteries or capillaries; it would be equivalent to give ergot in parturition when the uterus or its cervix or the perineum is in a state of spasm, or to give medicine to promote peristaltic action of the intestine when a section of it was locked in a spasm of cramp colic, or to give strychnin in tetanus. When the condition justifies its use, it is without a rival in improving the rhythm of the heart and blood vessels and in securing relief to the cardio-respiratory distress.

Strophanthus improves the systole and diastole of the heart similar to digitalis, but in less degree; it differs from it in not impressing the arteries, capillaries and veins; therefore, it is indicated when the heart needs a spur to improve the vigor of its action and the lumen of the blood vessels is not offering any resistanee.

Strychnin is another heart tonic, but it is so only as it improves the tonicity of the entire nervous system, and causes quick response in converting potential into kinetic dynamics; this is soon exhausted unless assim. ilative metabolism is sufficient to compensate the over-draught occasioned by the strychnin. These three remedies, strychnin, digitalis and strophanthus are invaluable cardio-vascular tonics with some latitude of difference in their therapeutic value; but when administered with proper discrimination of the patholgy involved, they make more stable the results obtained by the next class of remedies; ethyl nitrite (nitrous ether), morphin and atropia, nitrite of amyl, nitrite of sodium, nitroglycerin, methylene-blue, hyoscin hydrobromate, etc. These fulminant remedies are complex in their molecular construction, unstable chemic equilibrium, and, notably, nitrogen is one of the elements present and the one that determines their wonderful physical and dynamic properties. These substances are remedies of great value, for quick results, in relaxing the high cardiac, arterial and capillary tension, whether it be that of systole, diastole or in statu quo. They reach their therapeutic virtue through the sensory pathway of the vagus, its ganglia and its center in the medulla. This constitutes one of the special distinctions of this class of remedies. Whenever there is a tonic or clonic spasm, whether it is in the heart and blood vessels, or the intestine, or any other viscus with muscular walls, relief comes from remedies that are analgesic (sensory-pathway). They are classed as stimulants to vaso-motor mechanism, but they are only so by unlocking the tonic muscular contractility, either systolic or inhibitory, through the sensory mechanism; then the rhythmic function is re-established. Nitroglycerin is possibly the best of the class, while not so prompt as nitrite of amyl, its effect is more enduring. A proper blending of the medicines above discussed, according to their therapeutic virtue, a general nerve tonic (strychnin), a vaso-motor tonic (digitalis) and the analgesic remedies (nitrite of amyl or nitroglycerin, etc.), give us theoretically, all that the most critical analysis of the indications involved and demanded in the treatment of the paroxysmal factor of idiopathic angina pectoris. I have at this time a patient, a woman aged 50 years, who at various times for the past ten years has been the subject of this disease. Her paroxysms realize all the characteristic symptoms, often with severe asthma and ataxia of the diaphragm, which has been relieved with morphia and atropia hypodermically; within the past 
month she was seized with one of her paroxysms but one more urgent and critical; I administered hypdermically, a combination dose of strychnin 1-40 gr., ntiroglycerin 1-100 gr., and digitalin 1-100 gr., obtaining relief and very much more satisfactory results than the morphia and atropia had previously given.

\section{ATROPHIC RHINITIS.}

CANDIDATE'S THESIS FOR THE AMERICAN LARYNGOLOGICAL ASSOCIATION. BY JOHN EDWIN RHODES, A.M., M.D.

Professor of Physical Diagnosis and Clinical Medicine Northwestern Cniversity Woman's Medical School: Lecturer on Laryngology and Diserses of the Chest, Rush Medical College; Fellow of the American Laryngological Association, etc.

Atrophic rhinitis is a chronic inflammation of the lining membrane of the nasal cavities resulting in atrophy of tissues, with occasional corresponding changes in the bony structure, and the formation of crusts. It is frequently accompanied by ozena.

While all authorities are practically agreed as to the final sclerosis of the tissues in atrophic rhinitis, there are differences of opinion among observers as to the exact pathologic process by which such a result has been brought about. Is the atrophic condition essentially such from the beginning or is it preceded by hypertrophy? Manifestly, opinion founded upon clinical observation must be surrounded with difficulties. Patients are usually seen during some stage of the process and are seldom under observation from the inception of the disease; often the disease has extended over many years and a satisfactory history of it during the early stages can not be obtained. Occasionally observers have been able to follow the process from its beginning. Thus Schäffer found cases in which there had been hypertrophy for eight or ten years which was followed by atrophy. It is not uncommon to find in one side of the nares, hypertrophy of the turbinates and in the other atrophy; there frequently is also within one nasal cavity hypertrophy of the middle turbinated with atrophy of the lower, or vice versa-a condition observed also by Capart $^{1}$ and others. Again, it has been common, on inquiry, to find that the obstruction of the nasal cavities was the predominant early symptom. In our histories of 142 cases of private patients, I find one in which we observed the process from its inception. The patient was a girl, aged 15. She was only treated at very long intervals, so this could have had no bearing on the result. In this case the hypertrophy.was well marked when the case first came under observation. Within two years atrophy had taken place, which subsequently became extreme. More ${ }^{2}$ marked the coincidence of atrophic and hypertrophic rhinitis in eighteen out of eighty cases. I have but recently seen a well-marked case in which there was hypertrophy of the inferior turbinated upon one side and atrophy well advanced upon the other. In our list of cases I find several in which similar conditions existed.

J. N. Mackenzie ${ }^{3}$ believes that in this form of rhinitis we have the concluding stage of nasal inflammation, and that it is usually the sequel of a hypertrophic condition. In support of his position he traces the pathologic processes from an intumescent condition, resulting in the temporary obstruction of one or both nares. At first there is an engorgement of tissues mainly confined to the erectile spaces, with perhaps a slight cellular infiltration of the submucous tissues and an unchanged epithelium. When permanent swelling of the membrane has taken place the intercellular walls are changed into a dense fibrous structure, encroaching more and more upon the erectile spaces, and a fibrous deposit in the submucous tissues compresses the glandular elements and gradually obliterates them. These conditions also invade the superficial layers, and the blood supply is interfered with, causing destruction of, or changes in the epithelial layer. In the obliteration of the erectile spaces he also observed in two specimens the presence in the spaces of masses of round cells; also in certain cases the formation of parietal thrombi and occasionally bulbous processes projecting from the walls of the cavernous sinuses. Finally the histologic condition seems to be a gradual atrophy of the different layers of the membrane, and as has been shown in the studies of Eugene Fränkel and others, we have presented to us a cirrhotic processs in which the mucous membrane shrinks away by being changed into a fibrous connective tissue.

Browne, ${ }^{4}$ while willing to accede to a quite generally antecedent hypertrophy, in which there is an increase of the connective tissue elements, which culminates in fibroid shrinking, is of opinion that there are cases in which the process is atrophic from the beginning. Seiler's views coincide with this statement. Sajous ${ }^{5}$ is satisfied that atrophy is an occasional result of hypertrophic rhinitis, and that the pressure of dried secretions on the membrane is an important factor in the production of atrophy. The pressure of dried secretions can not be a constant factor, because in some cases of atrophy the secretions are either fluid or are easily removed. Atrophy has already begun in many cases and proceeded to quite an advanced stage, in which dried secretions have not been a feature. Over much of the membrane, especially at the anterior portion of the external wall of the nares, the crusts in many cases have never formed. It does not seem possible that, frequently at any rate, there could be sufficient pressure to interfere in any marked degree with the vascularity of the tissues. The increase of the crust takes place upon the surface of the membrane, and as it increases it is forced further from the membrane and the extreme, dry, contracting portion is upon the external free surface of the crust, the under part being usually moist and semi-liquid.

Delsaux ${ }^{6}$ believes that a simple chronic rhinitis is the precursor of atrophy, and does not admit that hypertrophy always precedes it. Ingals ${ }^{7}$ favors the theory that in most cases atrophy is the result of a previous hypertrophy. B. Fränkel ${ }^{s}$ also states that in most cases atrophy is the result of a preceding hyper. plastic condition. Cohen ${ }^{9}$ affirms that in these cases we early find hypertrophy. A similar belief is expressed by M. Mackenzie. ${ }^{10}$ Abel $^{11}$ believes the bacillus mucosus capseolatus, causing a simple catarrhal inflammation. leads to primary hypertrophy and then to atrophy. On the other hand, Bosworth ${ }^{12}$ does not admit that hypertrophic rhinitis is in any case the cause of the atrophic, but that the morbid changes are atrophic from the outset. Capart ${ }^{13}$ also believes that the disease is atrophic from the begining and not the result of a preceding hypertrophy.

It was formerly quite generally believed that the syphilitic or strumous diatheses played an important rôle in the etiology of the disease. It is probable that these conditions may increase the susceptibility, and exercise some influence in its production, but the affection, in the majority of cases, develops in an otherwise perfectly healthy individual. 